\title{
Constraints on spinning dust towards Galactic targets with the Very Small Array: a tentative detection of excess microwave emission towards 3C396
}

\author{
Anna Scaife, ${ }^{1 \star}$ David A. Green, ${ }^{1}$ Richard A. Battye, ${ }^{2}$ Rod D. Davies, ${ }^{2}$ \\ Richard J. Davis, ${ }^{2}$ Clive Dickinson, ${ }^{3,6}$ Thomas Franzen, ${ }^{1}$ Ricardo Génova-Santos, ${ }^{1}$ \\ Keith Grainge, ${ }^{1}$ Yaser A. Hafez, ${ }^{2}$ Michael P. Hobson, ${ }^{1}$ Anthony Lasenby, ${ }^{1}$ \\ Guy G. Pooley, ${ }^{1}$ Nutan Rajguru, ${ }^{4}$ Rafael Rebolo, ${ }^{5}$ José Alberto Rubiño-Martin, ${ }^{5}$ \\ Richard D. E. Saunders, ${ }^{1}$ Paul F. Scott, ${ }^{1}$ David Titterington, ${ }^{1}$ Elizabeth Waldram ${ }^{1}$ \\ and Robert A. Watson ${ }^{2}$ \\ ${ }^{1}$ Astrophysics Group, Cavendish Laboratory, 19 J. J. Thomson Ave, Cambridge CB3 OHE \\ ${ }^{2}$ Jodrell Bank Observatory, Macclesfield, Cheshire SK11 9DL \\ ${ }^{3}$ Jet Propulsion Laboratory, 4800 Oak Grove Drive, M/S 169-327, Pasadena, CA 91109, USA \\ ${ }^{4}$ University College London, Department of Physics \& Astronomy, Astrophysics Group, Gower Street, London WC1E 6BT \\ ${ }^{5}$ Instituto de Astrofísica de Canarias, 38200 La Laguna, Tenerife, Spain \\ ${ }^{6}$ California Institute of Technology, Mail Code 105-24, Pasadena, CA 91125, USA
}

Accepted 2007 February 16. Received 2007 February 16; in original form 2006 November 30

\begin{abstract}
We present results from observations made at $33 \mathrm{GHz}$ with the Very Small Array (VSA) telescope towards potential candidates in the Galactic plane for spinning dust emission. In the cases of the diffuse H II regions LPH96 and NRAO591 we find no evidence for anomalous emission and, in combination with Effelsberg data at 1.4 and $2.7 \mathrm{GHz}$, confirm that their spectra are consistent with optically thin free-free emission. In the case of the infrared bright supernova remnant 3C396 we find emission inconsistent with a purely non-thermal spectrum and discuss the possibility of this excess arising from either a spinning dust component or a shallow spectrum pulsar wind nebula, although we conclude that the second case is unlikely given the strong constraints available from lower-frequency radio images.
\end{abstract}

Key words: radiation mechanisms: general - dust, extinction - ISM: individual: 3C396 radio continuum: ISM.

\section{INTRODUCTION}

A localized excess of emission in the microwave region was first detected in the $C O B E / \mathrm{DMR}$ data and was initially attributed to freefree emission (Kogut et al. 1996a,b). Since then this anomalous emission has been detected by a number of authors (de OlivieraCosta et al. 2002, 2004; Banday et al. 2003; Finkbeiner, Langston \& Minter 2004; Watson et al. 2005; Fernández-Cerezo et al. 2006) and has been nicknamed 'Foreground X'. Although initially ascribed to thermal bremsstrahlung in view of its strong correlation with thermal dust, low $\mathrm{H} \alpha$ surface brightness measurements (Leitch et al. 1997) implied gas temperatures in excess of $10^{6} \mathrm{~K}$, which were rejected on energetic grounds by Draine \& Lazarian (1998a). Its physical mechanism has yet to be constrained; the most popular interpretation is that of rapidly rotating dust grains, or spinning dust (Draine \& Lazarian 1998a,b). Other mechanisms which have been

${ }^{\star}$ E-mail: as595@mrao.cam.ac.uk proposed include magnetic dust emission (Draine \& Lazarian 1999), flat spectrum synchrotron (Bennett et al. 2003), and bremsstrahlung from very hot electrons (Leitch et al. 1997). Spinning dust causes an excess of emission in the $10-50 \mathrm{GHz}$ region of the spectrum, where the combination of synchrotron, bremsstrahlung and thermal dust emission is a minimum, and this is problematic especially for cosmic microwave background (CMB) experiments which utilize this region to minimize foreground contamination and avoid atmospheric emission. Consequently the presence of a poorly constrained foreground such as this anomalous dust emission is a potentially serious problem for CMB observers and needs to be better understood in order to be correctly removed. This has led to several directed observations (Finkbeiner et al. 2004; Watson et al. 2005; Casassus et al. 2004, 2006; Dickinson et al. 2006) being made towards targets suggested by the theoretical predictions of Draine \& Lazarian (1998a,b, hereinafter DL98b). Whilst these have been mainly directed at $\mathrm{H}$ II regions and dark clouds, DL98b also suggest that it may be possible to detect $10-100 \mathrm{GHz}$ emission from spinning dust in photodissociation regions. Here we present observations at $33 \mathrm{GHz}$ towards two 
$\mathrm{H}$ II regions and one supernova remnant (SNR) for which infrared observations imply the presence of significant photodissociation regions (PDRs).

\section{THE TELESCOPE}

The VSA is a 14-element interferometer sited at the Teide Observatory, in Tenerife, at an altitude of $2400 \mathrm{~m}$. The VSA operates in a single $1.5-\mathrm{GHz}$-wide channel at a central frequency of $33 \mathrm{GHz}$ (Scott et al. 2003). The 14 antennas use HEMT amplifiers with typical system temperature $\approx 35 \mathrm{~K}$. In its new super-extended configuration the VSA uses mirrors of diameter $65 \mathrm{~cm}$. The horn-reflector antennas are mounted on a tilt table hinged east-west and each antenna individually tracks the observed field by rotating its horn axis perpendicularly to the table hinge, wavefront coherence being maintained with an electronic path compensator system (Watson et al. 2003).

The individual tracking of the VSA antennas allows for the filtering of contaminating signals. These may be celestial sources, such as the Sun and Moon, or ground-spill and other environment-based spurious signals. The VSA also uses a ground-shield to minimize ground-spill. The consequences of this design are two-fold. First, the VSA is able to observe continuously and can filter out emission from the Sun and Moon when they are as close as $9^{\circ}$. Secondly, the VSA is unaffected by ground-spill contamination for fields within $35^{\circ}$ of the zenith and so is able to make direct images of the sky, rather than employing the lead-trail approach of many other interferometers operating in the microwave band.

\section{OBSERVATIONS}

Observations were carried out as single pointings with a primary beam of 72-arcmin full width at half maximum (FWHM). The data were calibrated using Tau A and Cas A in accordance with VSA reduction procedures. This calibration, along with appropriate flagging and filtering of the data, was performed using the special purpose package REDUCE developed specifically for reduction of VSA observations. Details of the VSA reduction and calibration procedure can be found in Dickinson et al. (2004) and references therein.

Many surveys at lower frequency do not have similar resolution to the super-extended VSA, making comparison difficult. Instead we extrapolate from the Effelsberg 100-m telescope at $2.7 \mathrm{GHz}$ (Fürst et al. 1990a). These data ${ }^{1}$ are single-dish maps with a circular beam of 4.3-arcmin FWHM.

For a robust comparison, the 2.7-GHz Effelsberg maps were multiplied by the primary beam of the VSA and visibility sampling was performed in the $u v$-plane with a correction for the Effelsberg beam.

\section{LPH96}

The H II region LPH96 $\left(\mathrm{RA}=06^{\mathrm{h}} 36^{\mathrm{m}} 40^{\mathrm{s}}, \delta=+10^{\circ} 46^{\prime} 28^{\prime \prime}, \mathrm{J} 2000\right)$ has been observed with the Green Bank 43-m telescope between 5 and $10 \mathrm{GHz}$ (Finkbeiner et al. 2002) and was shown to have a rising spectrum consistent with that expected from spinning dust. However, a pointed observation made with the Cosmic Background Imager (CBI) telescope (Dickinson et al. 2006) at $31 \mathrm{GHz}$ shows emission consistent with an approximately flat spectrum source with only little possibility of spinning dust.

\footnotetext{
${ }^{1}$ The Effelsberg survey data were downloaded from the MPIfR sampler
} survey website: http://www.mpifr-bonn.mpg.de/survey.html
At $33 \mathrm{GHz}$ the peak flux density of LPH96 is $1.100 \pm 0.030 \mathrm{Jy}$ beam ${ }^{-1}$. The synthesized beam of the VSA towards LPH96 is $9.1 \times$ $6.3 \operatorname{arcmin}^{2}$ and the source is slightly resolved with structure extending towards the north and south-west. The $2.7-\mathrm{GHz}$ data after sampling gives a peak flux density of $1.436 \pm 0.010 \mathrm{Jy} \mathrm{beam}^{-1}$. Comparing this with the VSA flux density we find a spectral index $\alpha=0.106 \pm 0.026$, where the errors are calculated from the thermal noise outside the beam on the 2.7- and 33-GHz maps. The spectral index, $\alpha$, is here defined so that flux density scales as $v^{-\alpha}$. This calculation fails to take into account systematic errors; taking errors of 5 per cent on the flux density scales at each frequency gives $\alpha=$ $0.106 \pm 0.065$.

The VSA measurement shows no indication of the excess emission which would be expected at $33 \mathrm{GHz}$ from the warm neutral medium (WNM) spinning dust model of DL98b consistent with the Green Bank data. The result is more consistent with that found using the CBI telescope (Dickinson et al. 2006); it was found that emission at $31 \mathrm{GHz}$ is consistent only with $\alpha=0.06 \pm 0.03$. Dickinson et al. also note that the Galactic plane survey of Langston et al. (2000) failed to detect LPH96 at $14.35 \mathrm{GHz}$ with a detection limit of $2.5 \mathrm{Jy}$.

\section{$5 \mathbf{3 C 3 9 6}$}

The SNR 3C396 (= G39.2-0.3) is a shell-like remnant at radio frequencies, with a mean angular diameter of 7.8 arcmin (Patnaik et al. 1990). Its spectral behaviour has been extensively studied in the radio, most notably by Patnaik, and has a non-thermal radio spectrum with $\alpha \approx 0.42$ between $\sim 400 \mathrm{MHz}$ and $\sim 10 \mathrm{GHz}$. Below $30 \mathrm{MHz}$ catalogued flux densities for 3C396 are contaminated by the nearby steep-spectrum pulsar, PSR 1900+0.5 (Manchester \& Taylor 1981). A pulsar wind nebula (PWN) near the centre of this SNR has been detected in X-rays (see Olbert et al. 2003), but the remnant has not been detected optically. Only a lower limit of $7.7 \mathrm{kpc}$ for its distance is available from its $\mathrm{HI}$ absorption observation (Caswell et al. 1975). Patnaik et al. conclude that the neighbouring $\mathrm{H}$ II region NRAO 591 is likely to be at a distance of $\simeq 14 \mathrm{kpc}$.

\subsection{VSA observation of $3 \mathrm{C} 396$}

3C396 was observed in 2006 August in a short observation of $1.8 \mathrm{~h}$. Mapping and clean-based deconvolution were performed using the AIPS package. The observation was not limited by thermal noise but rather by the dynamic range of the telescope, and it has an rms noise of $34.0 \mathrm{mJy}$. The VSA map is shown in Fig. 1; it shows both 3C396 and also the HII region NRAO 591 to the north-west of the remnant. The VSA beam towards 3C396 is $9.1 \times 7.7 \mathrm{arcmin}^{2}$. Interesting features include a protrusion to the north and a faint detection of the 'blow-out' tail to the north-east, both of which are also present at longer radio wavelengths.

Fits were obtained using the AIPS task JMFIT by drawing a bounding box around both sources and fitting for two Gaussians and a base level. The peak flux density for 3C396 was found to be $3.21 \pm$ $0.29 \mathrm{Jy} \mathrm{beam}^{-1}$ and the integrated flux density $6.64 \pm 0.33 \mathrm{Jy}$ at $33 \mathrm{GHz}$, with the peak at $19^{\mathrm{h}} 01^{\mathrm{m}} 43^{\mathrm{s}} .2+05^{\circ} 22^{\prime} 22^{\prime \prime} .4$ (B1950). For the secondary source NRAO 591 we find a peak flux density of $1.71 \pm 0.09 \mathrm{Jy} \mathrm{beam}^{-1}$ and an integrated flux density of $2.40 \pm$ 0.12 Jy centred at $19^{\mathrm{h}} 00^{\mathrm{m}} 47^{\mathrm{s}} .4+05^{\circ} 31^{\prime} 06^{\prime \prime} \cdot 3$ (B1950). The errors here include contributions from the rms noise on the observation, the statistical error from the Gaussian fits, and a conservative 5 per cent error from the flux calibration which dominates the overall 


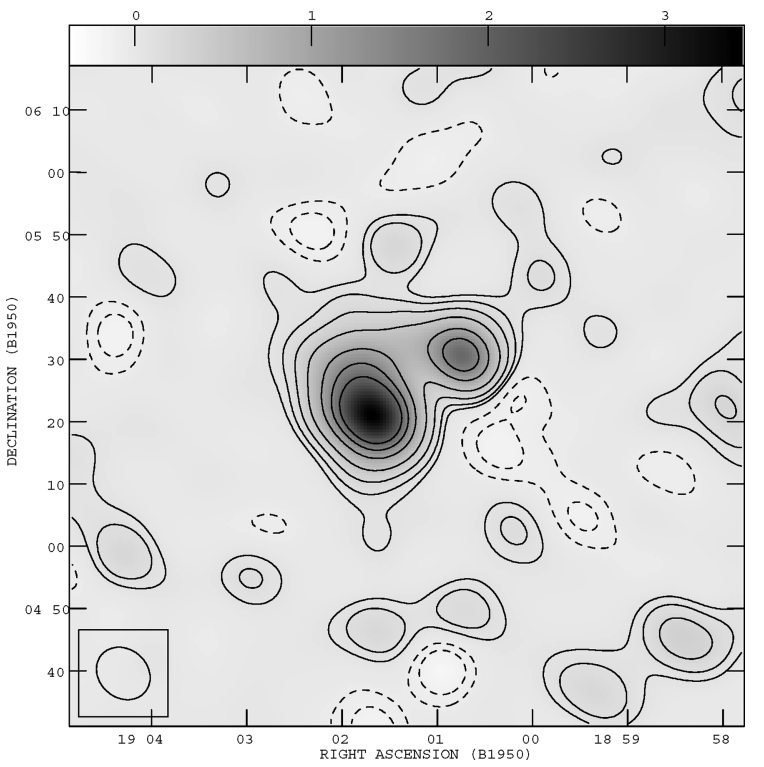

Figure 1. VSA observation of $3 \mathrm{C} 396$ with the $\mathrm{H}$ II region NRAO 591 also visible to the north-west. Contours are overlaid at $-2,-1,1,2,4,8,16,32$ and $64 \sigma$.

value. A complete discussion of the VSA flux calibration may be found in Dickinson et al. (2004).

\subsection{The integrated spectrum of $3 \mathrm{C396}$}

Again we use observations from the Effelsberg 100-m telescope to examine the emission at lower radio frequencies. Using Effelsberg data at 1.4 (Reich, Reich \& Fürst 1997) and 2.7 GHz, we convolve the data at $2.7 \mathrm{GHz}$ to match the $1.4-\mathrm{GHz}$ resolution of $9.4 \mathrm{arcmin}$ and find flux densities of $14.9 \pm 1.3 \mathrm{Jy}$ and $11.4 \pm 1.3 \mathrm{Jy}$ at 1.4 and $2.7 \mathrm{GHz}$, respectively. These values agree with those of Reich, Reich \& Fürst (1990) and give a spectral index of $\alpha=0.46$, which is consistent with the mean of 0.45 found for shell-type SNRs (Green 2004, 2006). After sampling the Effelsberg observation at $2.7 \mathrm{GHz}$ to match the $u v$-coverage of the superextended VSA towards 3C396 we find a flux density of $10.90 \pm 0.51 \mathrm{Jy}$. Our best-fitting index implies a flux density of $3.4 \mathrm{Jy}$ at $33 \mathrm{GHz}$ extrapolated from the Effelsberg data.

We investigate the spectrum of 3C396 using our own flux density and other published values; all errors are quoted to $1 \sigma$. Taken at face value the VSA observation of 3C396 would imply an index $\alpha_{33}^{2.7}=$ $0.20 \pm 0.02$, broadly consistent with a region of thermal emission. Alternatively, if we assume the index $\alpha_{2.7}^{1.4}$ of 0.46 determined from the Effelsberg data, then the VSA flux density would imply an excess of emission seen at microwave frequencies towards this source. A large number of observations between $400 \mathrm{MHz}$ and $5 \mathrm{GHz}$ exist and to confirm this spectral index we compile a spectrum using flux densities taken from Patnaik et al. (1990) who made corrections to the original measurements to bring them on to the flux density scale of Baars et al. (1977). The integrated spectrum of 3C396 is shown in Fig. 2. Data from Patnaik et al. is shown as crosses, the data from Effelsberg as filled squares and the VSA data as an unfilled diamond. Performing a weighted least-squares fit to the Patnaik and Effelsberg data we find an index of $\alpha=0.42 \pm 0.03$. The VSA measurement at $33 \mathrm{GHz}$ is inconsistent with this spectrum, shown in Fig. 2 as a solid line. Including the VSA data at $33 \mathrm{GHz}$ we find a spectral index of $\alpha=0.32 \pm 0.02$, shown as a dashed line.

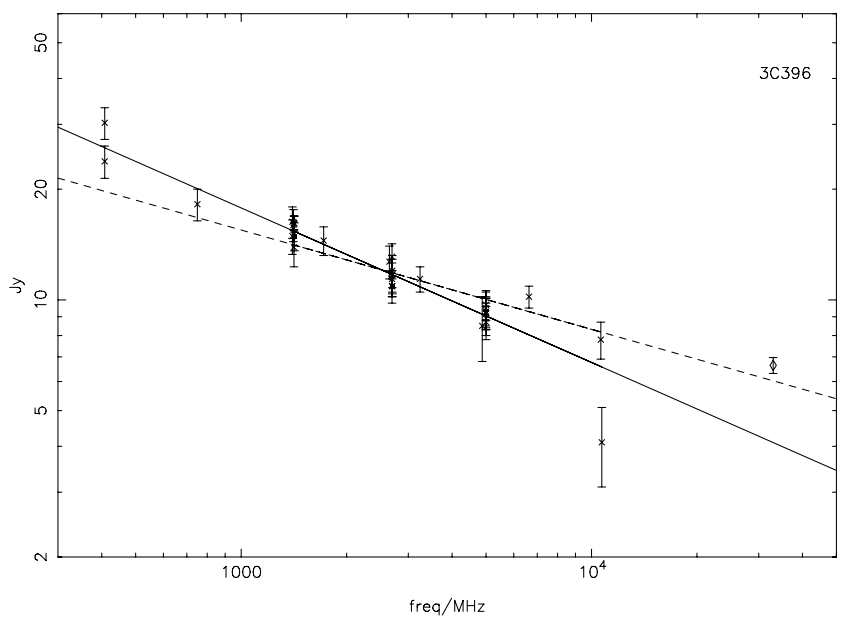

Figure 2. Integrated spectrum of 3C396. Data points are taken from Patnaik et al. (1990) with the exception of Effelsberg measurements at 1.4 and $2.7 \mathrm{GHz}$ which are indicated by filled squares. A spectral index of $\alpha=0.42$ is shown as a solid line and of $\alpha=0.32$ as a dashed line. The measurement from the VSA at $33 \mathrm{GHz}$ is shown as an unfilled diamond.

However, $\chi^{2}$ values for the two fitted spectra show that the spectral index of $0.42\left[\chi_{\text {red }}^{2}=1.07,31\right.$ dof, $\left.\mathrm{P}(1.07)=0.366\right]$ is a better fit to the data. Indeed a spectral index of $0.32\left[\chi_{\text {red }}^{2}=1.95,32\right.$ dof, $\mathrm{P}(1.95)=0.001]$ would be unusually flat for a supernova remnant with less than 7 per cent of shell-like or possible shell-like supernova remnants having a spectral index of less than or equal to 0.32 (Green 2006). However, we hesitate to overinterpret this statistic because the catalogued spectral indices are by no means uniform in quality.

Because previous observations (Finkbeiner et al. 2004) have suggested the presence of spinning dust in $\mathrm{H}$ II regions, we have also compiled a spectrum for NRAO 591. We use flux densities compiled by Patnaik et al. (1990) which are plotted in Fig. 3 and find a spectrum compatible with that of optically thin free-free emission.

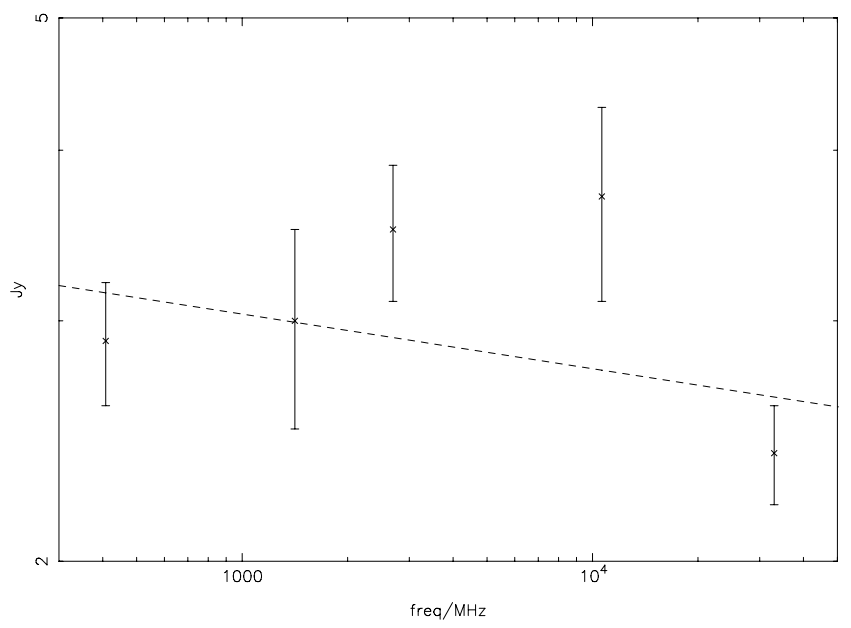

Figure 3. Integrated spectrum of NRAO 591. Fluxes are taken from Patnaik et al. (1990) with the exception of the VSA data point at $33 \mathrm{GHz}$ which is shown as a diamond. A best-fitting spectral index of $\alpha=0.05 \pm 0.06$ is shown as a dashed line. 


\subsection{Discussion and conclusions}

For the source 3C396 we constrain the possible excess emission relative to the non-thermal contribution at $33 \mathrm{GHz}$ by subtracting a non-thermal model extrapolated from lower frequency. For the flux densities between $400 \mathrm{MHz}$ and $10 \mathrm{GHz}$, shown in Fig. 2, a bestfitting spectral index of $\alpha=0.42 \pm 0.03$ was found. This index implies a flux density of $4.19 \pm 0.11 \mathrm{Jy}$ at $33 \mathrm{GHz}$, where the error is statistical only. This leaves $2.45 \pm 0.35 \mathrm{Jy}$ that may be due to an anomalous component. If we include a 5 per cent error on the flux calibration these errors are increased to 0.24 and $0.41 \mathrm{Jy}$, respectively. The excess accounts for 37 per cent of the total $33 \mathrm{GHz}$ flux density.

Radio recombination line observations (Anantharamaiah 1985) put an upper limit on the emission measure of $3 \mathrm{C} 396$ of $280 \mathrm{~cm}^{-6} \mathrm{pc}$ and fits an electron temperature of $5000 \mathrm{~K}$ for the gas. This implies an upper limit on the free-free emission from the SNR of $0.061 \mathrm{Jy}$ beam $^{-1}$ at $33 \mathrm{GHz}$. The beam sizes used for this measurement are poorly matched to that of the VSA and may cause the values to be overestimated. Taking this into account it can be seen that the free-free contribution is small compared to the non-thermal and can only account for $\sim 2$ per cent of the excess emission. In addition, models including both free-free and non-thermal contributions provide poor fits to the Patnaik et al. data.

The possibility of the VSA seeing a secondary radio source in projection towards 3C396 is small. Radio sources with flux densities $>100 \mathrm{mJy}$ are seen with a frequency of $0.2 \mathrm{deg}^{-2}$. The possibility of a rising spectrum source at $>5 \mathrm{GHz}$ reduces this number by a factor of 10 (Waldram et al. 2003; Cleary et al. 2005).

Olbert et al. (2003) report the presence of a small PWN within the 3C396 SNR, although the authors note the lack of any corresponding radio feature in high-resolution 20-cm VLA images of the remnant (Dyer \& Reynolds 1999). For this PWN (or plerion-like component), to account for the excess flux density seen at $33 \mathrm{GHz}$ it would be very obvious at $1.4 \mathrm{GHz}$, where it would have to contribute approximately $1 / 6$ of the total flux density, assuming a flat spectral index - Olbert et al. however suggest that its contribution is $\leqslant 1 / 25$ of the total radio flux density at $1.4 \mathrm{GHz}$.

The infrared emission at $100 \mu \mathrm{m}$ towards 3C396 is shown in Fig. 4. In this region of the Galactic plane it is not certain whether the emission is associated with the SNR remnant or is merely a projection. Reach et al. (2006) suggest that higher-resolution Spitzer data shows emission at 3.6 to $8 \mu \mathrm{m}$ which may be associated with the SNR. They find IRAC colours for these regions consistent with both PDRs and $\mathrm{H}$ II regions.

If the excess emission seen at $33 \mathrm{GHz}$ from $3 \mathrm{C} 396$ is associated with these regions then it is possible that it may arise from the dipole emission of Draine \& Lazarian (1998a, DL98b). However, the discrepancy in resolution between the Spitzer and VSA telescopes precludes a more detailed spatial analysis. This possibility is illustrated in Fig. 5, where the data is shown with the WNM spinning dust model of DL98b. For clarity we have binned the data of Patnaik et al. at similar frequencies, and have excluded one point at $10.7 \mathrm{GHz}$. In addition, we note that the WNM model of DL98b, where the column density towards 3C396 is determined from the full-sky magnitude map of Schlegel, Finkbeiner \& Davis (1998), predicts a peak flux density at $33 \mathrm{GHz}$ of $3.5 \mathrm{Jy} \mathrm{beam}^{-1}$ at the resolution of the VSA due to spinning dust. This gives an integrated flux density of approximately $7 \mathrm{Jy}$, using the ratio of peak to integrated flux found with the VSA. However, it is likely that this method overestimates the integrated flux density because the morphology of the thermal dust emission appears more compact than the emis-

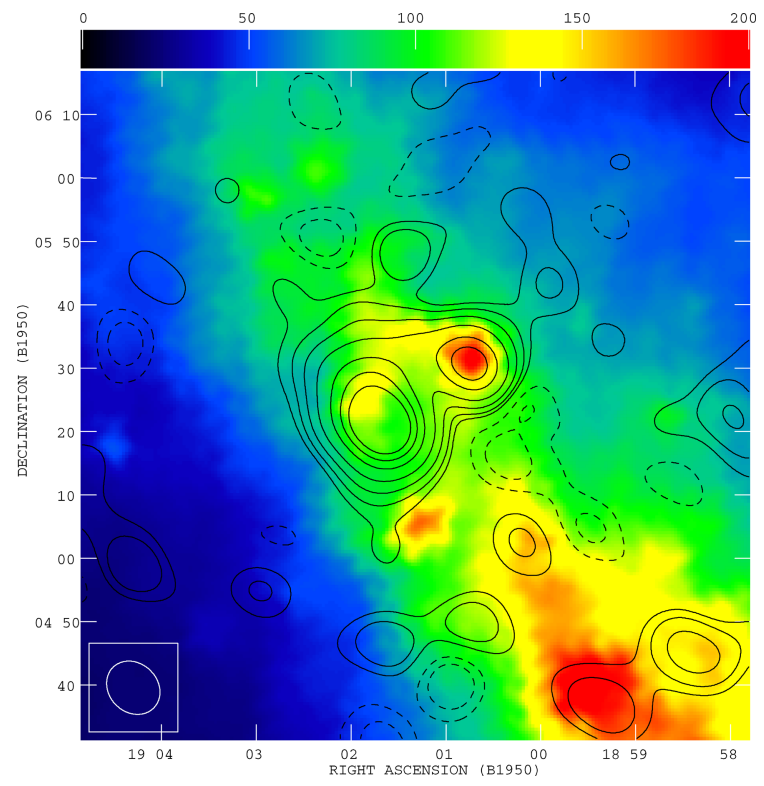

Figure 4. Infrared emission towards 3C396. Colour-scale is IRAS 100- $\mu \mathrm{m}$ data overlaid with the VSA observation at $33 \mathrm{GHz}$. Contours are $-2,-1,1$, $2,4,8,16,32$ and $64 \sigma$.

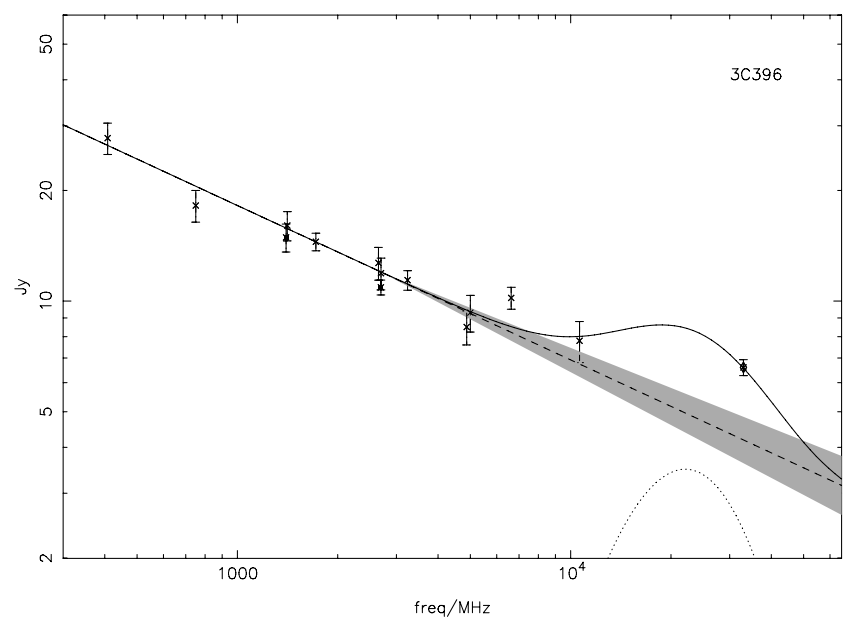

Figure 5. Integrated spectrum of 3C396. Data points from Patnaik and Effelsberg are as in Fig. 3 with data binned at similar frequencies. The best-fitting spectral index of $\alpha=0.42 \pm 0.03$ is shown as a dashed line and a spinning dust model (DL98b) scaled to fit the VSA data at $33 \mathrm{GHz}$ is shown as a dotted line. The measurement from the VSA at $33 \mathrm{GHz}$ is shown as an unfilled diamond.

sion seen with the VSA. It is however within a factor of 3 of the excess emission we see at $33 \mathrm{GHz}$.

In conclusion, we have assessed the possibility of spinning dust emission at $33 \mathrm{GHz}$ towards the SNR 3C396. Apart from Cas A and Tau A, few SNR have been studied in the microwave region. Consequently, in order to confirm this possibility further measurements are required in the range $10-20 \mathrm{GHz}$.

\section{ACKNOWLEDGMENTS}

We thank the staff of the Mullard Radio Astronomy Observatory, the Teide Observatory and the Jodrell Bank Observatory for their invaluable assistance in the commissioning and operation of the VSA. 
The VSA is supported by PPARC and the IAC. AS acknowledges the support of a PPARC studentship.

Part of the research described in this Letter was carried out at the Jet Propulsion Laboratory, California Institute of Technology, under a contract with the National Aeronautics and Space Administration.

We would also like to thank the anonymous referee for his careful reading of this Letter.

\section{REFERENCES}

Anantharamaiah K. R., 1985, J. Astrophys. Astron., 6, 177

Banday A. J., Dickinson C., Davies R. D., Davis R. J., Górski K. M., 2003, MNRAS, 345, 897

Baars J. W. M., Genzel R., Pauliny-Toth I. I. K., Witzel A., 1977, A\&A, 61, 99

Bennett C. L. et al., 2003, ApJS, 148, 97

Casassus S., Readhead A. C. S., Pearson T. J., Nyman L.-L., Shepherd M. C., Bronfman L., 2004, ApJ, 603, 599

Casassus S., Cabrera G. F., Förster F., Pearson T. J., Readhead A. C. S., Dickinson C., 2006, ApJ, 639, 951

Caswell J. L., Murray J. D., Roger R. S., Cole D. J., Cooke D. J., 1975 , A\&A, 45, 239

Cleary K. A. et al., 2005, MNRAS, 360, 340

de Oliviera-Costa A. et al., 2002, ApJ, 567, 363

de Oliviera-Costa A., Tegmark M., Davies R. D., Gutiérrez C. M., Lasenby

A. N., Rebolo R., Watson R. A., 2004, ApJ, 606, L89

Dickinson C. et al., 2004, MNRAS, 353, 732

Dickinson C., Casassus S., Pineda J. L., Pearson T. J., Readhead A. C. S. Davies R. D., 2006, ApJ, 643, L111

Draine B. T., Lazarian A., 1998a, ApJ, 494, L19

Draine B. T., Lazarian A., 1998b, ApJ, 508, 157 (DL98b)

Draine B. T., Lazarian A., 1999, ApJ, 512, 740

Dyer K. K., Reynolds S. P., 1999, AAS, 31, 973

Fernández-Cerezo S. et al., 2006, MNRAS, 370, 15
Finkbeiner D. P., Schlegel D. J., Frank C., Heiles C., 2002, ApJ, 566, 898

Finkbeiner D. P., Langston G. I., Minter A. H., 2004, ApJ, 617, 350

Fürst E., Reich W., Reich P., Reif K., 1990a, A\&AS, 85, 691

Fürst E., Reich W., Reich P., Reif K., 1990b, A\&AS, 85, 805

Green D. A., 2004, BASI, 32, 335

Green D. A., 2006, A Catalogue of Galactic Supernova Remnants (2006 April version). Astrophysics Group, Cavendish Laboratory, Cambridge, http://www.mrao.cam.ac.uk/surveys/snrs/

Kogut A., Banday A. J., Bennett C. L., Górski K. M., Hinshaw G., Reach W. T., 1996a, ApJ, 460, 1

Kogut A., Banday A. J., Bennett C. L., Górski K. M., Hinshaw G., Smoot G. F., Wright E. L., 1996b, ApJ, 464, L5

Langston G., Minter A., D’Addario L., Eberhardt K., Koski K., Zuber J., 2000, AJ, 119, 2801

Leitch E. M., Readhead A. C. S., Pearson T. J., Myers S. T., 1997, ApJ, 486, L23

Manchester R. N., Taylor J. H., 1981, AJ, 86, 1953

Olbert C., Keohane J. W., Arnaud K. A., Dyer K. K., Reynolds S. P., SafiHarb S., 2003, ApJ, 592, L45

Patnaik A. R., Hunt G. C., Salter C. J., Shaver P. A., Velusamy T., 1990 A\&A, 232, 467

Reach W. T. et al., 2006, AJ, 131, 1479

Reich W., Reich P., Fürst E., 1990a, A\&AS, 83, 539

Reich W., Fürst E., Reich P., Reif K., 1990b, A\&AS, 85, 633

Reich P., Reich W., Fürst E., 1997, A\&AS, 126, 413

Schlegel D. J., Finkbeiner D. P., Davis M., 1998, ApJ, 500, 525

Scott P. F. et al., 2003, MNRAS, 341, 1076

Waldram E. M., Pooley G. G., Grainge K. J. B., Jones M. E., Saunders R. D. E., Scott P. F., Taylor A. C., 2003, MNRAS, 342, 915

Watson R. A. et al., 2003, MNRAS, 341, 1057

Watson R. A. et al., 2005, ApJ, 624, L89

This paper has been typeset from a TEX/LATEX file prepared by the author. 